Bull. Mater. Sci., Vol. 3, Number 1, February 1981, pp. 25-28. Printed in India.

\title{
On the defect states in As-Se glasses*
}

\author{
R MOHAN and K J RAO \\ Solid State and Structural Chemistry Unit, Indian Institute of Science, \\ Bangalore 560 012, India
}

MS received 7 January 1981

\begin{abstract}
Magnetic susceptibility and ESR studies of As-Se glasses indicate that these glasses are diamagnetic containing only charged defect pairs and that they correspond to a chemically ordered network structure.
\end{abstract}

Keywords. As-Se glasses; magnetic susceptibilities; charged defect pairs.

\section{Introduction}

As a part of extensive investigations of As-Se glasses, we have now examined the nature of bonding and carrier states in As-Se glasses through magnetic susceptibility and ESR studies. Literature survey indicates that a few chalcogenide glasses show ESR absorption at $g \sim 2.0$ (Agarwal 1973; Kumeda et al 1976). ESR absorptions have however been attributed to either the method of preparation or to the impurities. Kumeda et al (1976) ascribed it to the effect of impurities which influence the creation of dangling bonds. We report in this communication ESR and magnetic susceptibility measurements on As-Se glasses which indicates that the maximum density of unpaired spins is less than $10^{16} \mathrm{~cm}^{-8}$ and hence the defects are present largely as charged diamagnetic centres. It also appears from susceptibility measurements that these glasses possess a chemically ordered network structure.

\section{Experimental}

These glasses were prepared from $5 \mathrm{~N}$ purity elements (Koch Light Company Buckinghamshire, UK) by melting in quartz, sealed under vacuum and agitating the melt for $12 \mathrm{hr}$ in a rotary furnace at $1000^{\circ} \mathrm{C}$ (Mohan et al 1980). Magnetic susceptibilities were measured using a Faraday set-up (Madhusudan 1980) and over a sufficiently wide temperature range. The sensitivity of the balance corresponds to $0.5 \%$ of $\chi_{M}$. ESR spectra were recorded using a Varian

* Communication No. 106 from Solid State and Structural Chemistry Unit. 
E109 spectrometer at room temperature and at a very high gain. The sensitivity of the instrument was estimated at $2-5 \times 10^{16} \mathrm{spins} / \mathrm{cm}^{3}$. The spectra were corrected for the spectrum from empty cavity.

\section{Results and discussion}

The magnetic susceptibilities of various compositions are shown as a function of temperature in figure 1 . It may be noted that these glasses are diamagnetic and that the susceptibilities are insensitive to temperature upto their glass transition temperatures. The molar susceptibilities $\left(\chi_{u}\right)$ for these samples can be calculated for a general composition $\mathrm{As}_{q} \mathrm{Se}_{\text {g }}$ by using the formula

$$
\chi_{H}=\chi_{\theta} M_{\mathrm{As}_{z} \mathrm{~S}_{\vartheta}}
$$

$\chi_{u}$ for $\mathrm{As}_{\boldsymbol{e}} \mathrm{Se}_{y}$ glasses can be computed by assuming two different models, namely, chemically ordered network model (CONM) and randomly ordered network model (RONM) as elaborated in earlier publications (Mohan et al 1980; Rao and Mohan 1980a). Chemically ordered network assumes the presence of $\mathrm{As}_{2} \mathrm{Se}_{2}$ plus excess element (Se or As) due to the preponderance of heteroatom bonds and the random ordered network has no such restriction. The diamagnetic susceptibilities can thus be calculated as

$$
\text { and } \quad \begin{aligned}
& \chi_{\mathrm{CONM}}=f \chi_{\mathrm{As}_{3} \mathrm{So}_{3}}+(1-f) \chi_{\mathrm{S}_{0} / \mathrm{Ass}} \\
& \chi_{\mathrm{RoNM}}=f^{\prime} \chi_{\mathrm{As}}+\left(1-f^{\prime}\right) \chi_{\mathrm{Se}_{0}}
\end{aligned}
$$

where $f$ is the mole fraction of $\mathrm{As}_{2} \mathrm{Se}_{3}$ in $\mathrm{As}_{4} \mathrm{Se}_{y}$ and is equal to $[x / 2(y-x)]$ in Se-rich glass and $[y /(3 x-y)]$ in As-rich glass. $f^{\prime}$ is simply the atomic fraction

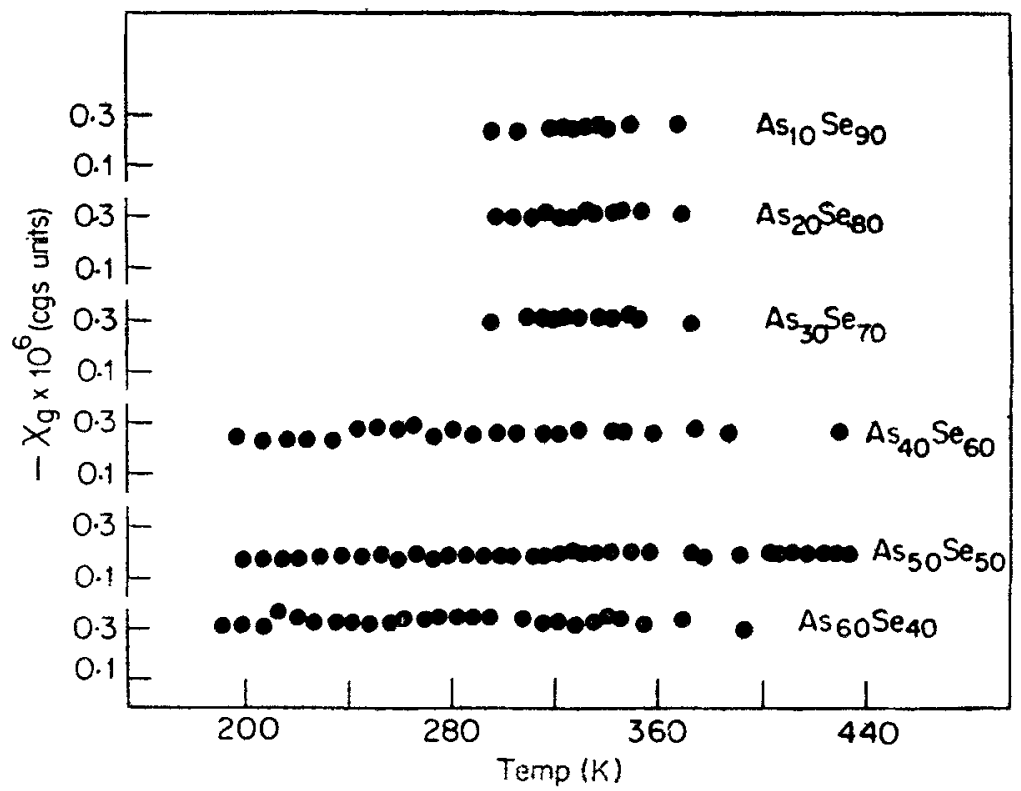

Figure 1. Variation of $\chi_{g}$ with temperature. 
of As corresponding to the same total weight of $\mathrm{As}_{a} \mathrm{Se}_{y}$ as in chemically ordered network model. The diamagnetic susceptibilities were evaluated using these formulae and reported values of diamagnetic susceptibilites. For $\mathrm{As}_{2} \mathrm{Se}_{3}$, we have taken the susceptibility measured in our experiments (for $\mathrm{As}_{40} \mathrm{Se}_{60}$ ). These values of $\chi_{\mu}$ are shown in figure 2. As-Se bonds are present in RONM structures also and are expected to make a contribution to their diamagnetic susceptibilities. However, equation (3) neglects any specific contribution due to $\mathrm{As}_{2} \mathrm{Se}_{3}$ type of units (and hence As-Se bonds) which are present in RONM structure. Figure 2 may be interpreted to support the presence of a chemically ordered network in As-Se glasses.

The ESR spectra are presented in figure 3. They demonstrate that the concentration of free spins corresponding to dangling bonds is negligible. However, a broad absorption centred around $g \sim 2.0$ is present particularly in Se-rich glasses. This may not be due to dissolved oxygen because we have observed in an earlier investigation (Mohan and Rao 1980) that the oxygen impurity is more likely to be present in As-rich side of these glasses. It is possible that they originate from the method of preparation as suggested by earlier workers (Moynihan et al 1975). An upper limit of about $10^{16} \mathrm{spins} / \mathrm{cm}^{3}$ which we estimated from these experiments (by comparison with signals obtained from spin probe doped glasses) is definitely 2-3 orders lower than the density of carriers indicated in our a.c. conductivity studies (Mohan et al 1980). Therefore these measurements very clearly support the view that most of the dangling bonds are involved in the formation of charged defect pairs. As we have shown elsewhere, these charged centres correspond to $\mathrm{Se}_{3}{ }^{+}$and $\mathrm{Se}_{1}-$ defects in As-Se system and their formation energies depend on the composition (Rao and Mohan 1980b).

Hence, the diamagnetic susceptibilities and ESR studies reported in this work indicate (i) the carriers are present primarily as diamagnetic charged defects of the type suggested by Kastner et al (1976) and (ii) that the structure of As-Se glasses corresponds more probably to a chemically ordered network structure.

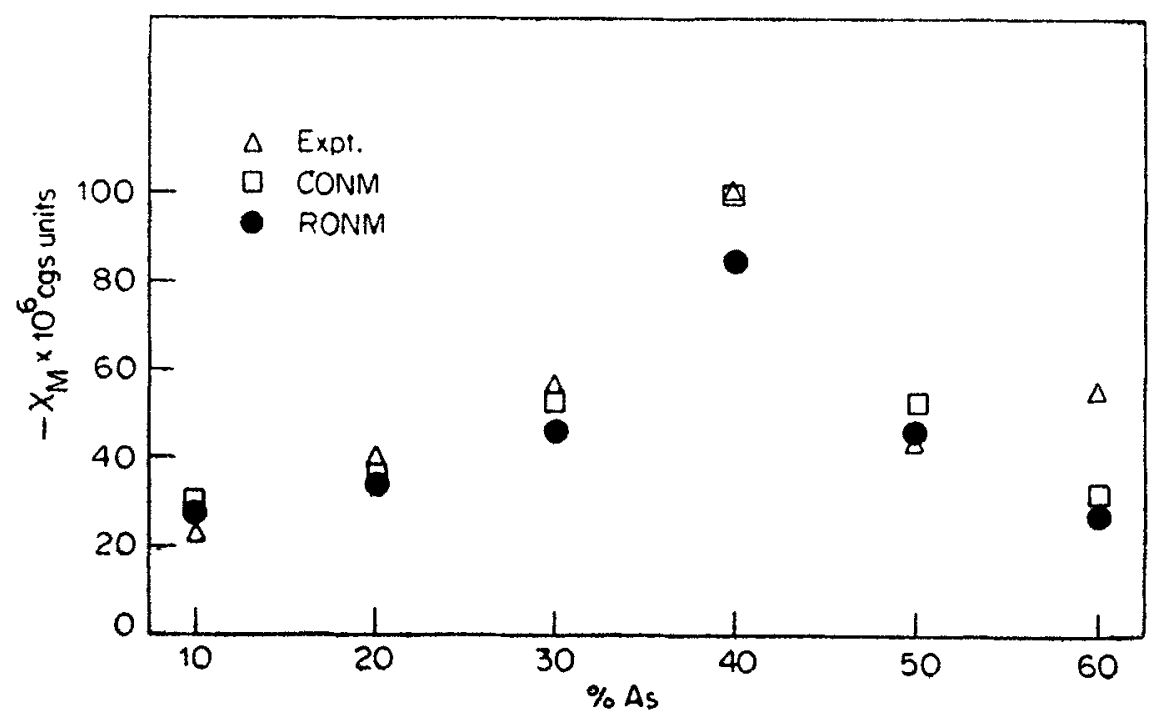

Figure 2. Variation of $\chi_{n}$ with composition. 


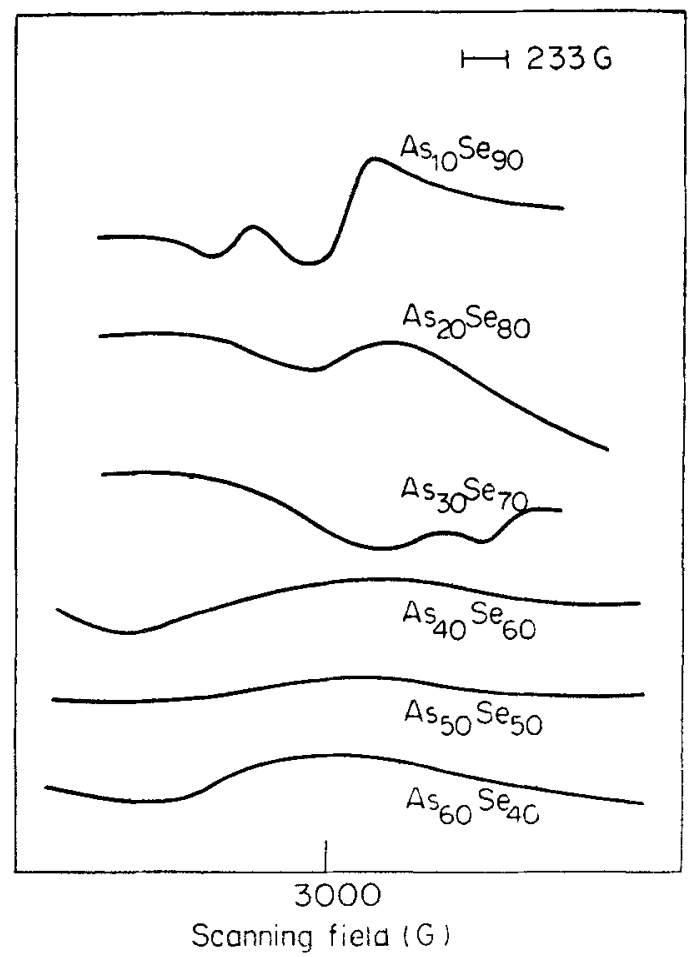

Figure 3. ESR spectra (from $1600 \mathrm{G}$ to $4600 \mathrm{G}$ ) of As-Se glasses.

\section{Acknowledgements}

The authors are very thankful to Professor C N R Rao of Indian Institute of Science for his kind encouragement. The authors thank the Department of Science and Technology, Government of India, for financial support.

\section{References}

Agarwal S G 1973 Phys. Rev. B7 685

Kastner M, Adler D and Fritzsche M 1976 Phys. Rev. Lett. 371504

Kumeda M, Kobayashi M, Maruyama E and Shimizu T 1976 Phys. Status Solidi B73 K19 Madhusudan W H 1980 X-ray spectroscopic and magnetic studies of transition metal oxide systems Ph.D. Thesis, Indian Institute of Science, Bangalore.

Mohan R, Sudha Mahadevan and Rao K J 1980 Mater. Res. Bull. 15917

Mohan R and Rao K J 1981 Proc. Indian Acad. Sci. (Chem. Sci.) Communicated

Moynihan C T, Macedo P B, Maklad M S, Mohr R K and Howard R E 1975 J. Non-Cryst. Solids 17369

Rao K J and Mohan R 1980a J. Phys. Chem. 841917

Rao K J and Mohan R 1980b Philos. Mag. (Communicated) 